\title{
JAK-2 V617F Mutational Analysis in Primary Idiopathic Myelofibrosis: Experience from Southern Pakistan
}

\author{
Sadia Sultan*, Syed Mohammed Irfan
}

\begin{abstract}
Background: Primary idiopathic myelofibrosis (PMF) is a clonal Ph-chromosome negative myeloproliferative neoplasm characterized by dysregulated kinase signaling and release of abnormal cytokines. In the recent past, following JAK2 V617F mutation invention, important revolution has been made in the molecular diagnostic biology of this disease. The rational of this study was to determine the mutational status of JAK2 V617F in Pakistan patients with PMF. Materials and Methods: In this cross sectional study, 20 patients with PMF were enrolled from January 2011 to December 2014. Diagnosis was based on WHO criteria for PMF. All patients were screened for G-T point mutation (V617F) in the JAK2 gene on chromosome 9 by allele specific PCR. Results: The mean age was $57.9 \pm 16.5 y$ ears. The male to female ratio was $3: 1$. The frequency of $J A K 2 \mathrm{~V} 617 \mathrm{~F}$ positivity in our PMF patients was found to be 55\%. Positive correlations of JAK2 V617F mutation were established with high TLC count, raised LDH and marked splenomegaly $(P<0.05)$. No correlation of JAK2 V617F could be established with age and gender $(\mathrm{P}>0.05)$. Conclusions: The JAK2 V617F mutation frequency in our PMF patients was similar to those reported previously. In our hands JAK2 V617F mutated patients expressed an aggressive disease phenotype. Screening for the mutation in all suspected PMF cases could be beneficial in differentiating patients with reactive and clonal marrow fibrosis.
\end{abstract}

Keywords: Primary idiopathic myelofibrosis - JAK-2 V617F - mutational analysis - Pakistan

Asian Pac J Cancer Prev, 16 (17), 7889-7892

\section{Introduction}

Myeloproliferative neoplasms (MPN) are a group of hematological neoplasms that share similar molecular, cellular and phenotypic abnormalities (Sag et al., 2015; Yang et al., 2015). Primary idiopathic myelofibrosis is a heterogeneous disease, characterized by dysregulated kinase signaling and release of abnormal cytokines resulting in debilitating constitutional symptoms, poor quality of life and high degree of morbidity.

The anticipated incidence of PMF is 0.5-1.5 per 100,000 individuals (Mesa et al., 1999; Tefferi., 2000). It occurs most commonly in the sixth to seventh decade of life and both genders are nearly equally affected (Thiele et al., 2008). The median age at diagnosis is around 66 years (Mitra et al., 2013).

In 2005, four research groups identified a novel single acquired mutation in the JAK2 gene on chromosome 9 which has been shown to be associated with a wide spectrum of chronic myeloproliferative neoplasm (Baxter et al., 2005; James et al., 2005).

This newly identified acquired somatic point mutation is a $\mathrm{G}-\mathrm{C}$ to $\mathrm{T}-\mathrm{A}$ transversion, resulting in the substitution of valine by phenylalanine at codon 617 (JAK2V617F) (Sazawal et al., 2010). JAK2 plays a role in downstream signaling pathways, such as the signal transducer and activator of transcription (STAT) pathway that is involved in cytokine signaling pathway.

$J A K 2 \mathrm{~V} 617 \mathrm{~F}$ has been recognized in subsets of each $\mathrm{Ph}$-chromosome negative MPNs. Different studies reported a variable prevalence of $J A K 2 \mathrm{~V} 617 \mathrm{~F}$ mutation ranging from 35-57\% for PMF (Poopak et al., 2013). JAK2 V617F mutation contributed to high hemoglobin, higher white blood cell count, larger spleen span and greater need for cytoreductive treatment (Barosi et al., 2007; Poopak et al., 2013; Liu et al., 2015).

Since, there is no study available from southern Pakistan; we sought to look for the prevalence of JAK2V617F mutation in Pakistani patients with primary myelofibrosis and also to determine its correlation with age, gender, hematological and biochemical parameters.

\section{Materials and Methods}

This descriptive cross sectional study, extended from January 2011 to December 2014. Twenty patients with primary idiopathic myelofibrosis were enrolled in the present study. An informed consent was obtained from all the participating patients.

Patients were diagnosed to have PMF according to the 
World Health Organization (WHO) criteria (Thiele et al., 2008). Diagnosis requires meeting all 3 major criteria and any of 2 minor criteria.

\section{Major criteria includes}

i) Presence of megakaryocyte proliferation and atypia, accompanied by fibrosis, or in the absence of fibrosis, the megakaryocyte changes must be accompanied by an increased bone marrow cellularity. ii) Not meeting WHO criteria for other myeloid neoplasm. iii) Demonstration of $J A K 2 \mathrm{~V} 617 \mathrm{~F}$ or in its absence no evidence of bone marrow fibrosis due to underlying inflammatory or other diseases.

\section{Minor criteria}

i) Leukoerythroblastic blood picture. ii) Raised serum lactate dehydrogenase level. iii) Anemia. iv) Splenomegaly.

Complete blood counts were determined by automated hematology analyzer Cell Dyne Ruby (Abott, Diagnostics). All peripheral blood smears were reviewed by specialist hematopathologists. Biochemical test including serum creatinine, lactate dehydrogenase (LDH) and serum uric acid were detected by Hitachi 912 (Japan) by photometric assay. JAK2 V617F mutational analyses were done by Polymerase chain reaction (PCR). Bone marrow aspirate and trephine biopsy specimen were taken with Jamshidi needle.

The ethical endorsement of the study was granted by research and ethical committee LNH taken prior to the study.

\section{Data analysis}

The demographic data, clinical characteristics, and laboratory results were analyzed by descriptive analysis. Data was compiled and analyzed using SPSS version 22. The results are expressed as mean \pm SD for quantitative variables and qualitative variables are presented as frequency \& percentages. Student ' $t$ ' test was applied for the comparison of mean. Data were considered statistically significant at $P$ value $<0.05$. Chi-square test was applied to assess the correlation.

\section{Results}

A total of 20 confirmed Primary idiopathic myelofibrosis patients using the non probability consecutive sampling were included in this study.

Out of 20 patients, 15 were males $(75 \%)$ and 5 were females $(25 \%)$ with male to female ratio of $3: 1$. The mean age was $57.90 \pm 16.51$ years (range 22-87) years with the median age of 60 years. Mostly patients (90\%) were symptomatic and presented with constitutional symptoms. In symptomatic patients, major complaints were weakness (80\%); weight lost (75\%); abdominal discomfort (60\%); night sweats (13\%); cardiovascular accident (5\%) and pruritus $(5 \%)$.

Physical examination revealed splenomegaly as predominant finding detected in 17 patients $(85 \%)$ with the mean splenic span of $22.17 \pm 2.04 \mathrm{~cm}$. The hepatomegaly was detected in $11(55 \%)$ patients followed by pallor in $7(35 \%)$ patients.
Table 1. Comparative Analysis of JAK-2 Mutation Positive and Negative Patients with Myelofibrosis

\begin{tabular}{lrrl}
\hline Parameters & $\begin{array}{c}\text { JAK-2 positive } \\
\text { mean(SD) } \\
\mathrm{n}=11\end{array}$ & $\begin{array}{c}\text { JAK-2 negative } \\
\text { mean(SD) }\end{array}$ & P-value \\
& \multicolumn{1}{c}{$\mathrm{n}=9$} & \\
\hline Age & $55.54(17.13)$ & $60.77(16.23)$ & 0.4 \\
Spleen span & $21.48(2.96)$ & $15.80(3.37)$ & $0.05^{*}$ \\
Hemoglobin & $9.32(2.5)$ & $8.93(2.7)$ & 0.7 \\
MCV & $90.21(21.09)$ & $85.84(19.35)$ & 0.6 \\
TLC count & $22.26(11.73)$ & $14.26(9.36)$ & $0.03^{*}$ \\
Platelets count & $287.81(261.16)$ & $470.50(379.12)$ & 0.2 \\
Uric acid & $4.0(1.14)$ & $6.3(1.8)$ & 0.3 \\
LDH & $840.0(26.7)$ & $622(33.7)$ & $0.02^{*}$ \\
Creatinine & $0.7(0.6)$ & $1.0(0.7)$ & 0.4 \\
\hline
\end{tabular}

*P-value statistically significant

The mean hemoglobin was $9.16 \pm 2.52 \mathrm{~g} / \mathrm{dl}$ with the mean MCV of $88.17 \pm 19.69 \mathrm{fl}$. The total leukocyte count of $17.63 \pm 19.17 \times 10^{9} / 1$ and platelets count were $346.50 \pm 321.93 \times 10^{9} / 1$. Overall $65 \%$ patients were anemic and $20 \%$ patients had thrombocytopenia. While leucopenia and leukocytosis were seen in $25 \%$ and $20 \%$ respectively. Serum lactate dehydrogenase, serum creatinine and uric acid were $731.0 \pm 154.1,0.82 \pm 0.22$ and $4.76 \pm 1.33$ respectively.

$J A K 2 \mathrm{~V} 617 \mathrm{~F}$ was found to be positive in $11(55 \%)$ patients, while $9(45 \%)$ were negative. The comparative analyses of $J A K 2 \mathrm{~V} 617 \mathrm{~F}$ positive and negative patients are shown in table-1.

No correlation could be established between JAK2 V617F positivity with age and gender $(\mathrm{P}>0.05)$. However comparative analysis revealed significantly high total leukocytic count of $22.26 \pm 11.73$ in $J A K 2 \mathrm{~V} 617 \mathrm{~F}$ positive patients group as compared with negative group that was $14.26 \pm 9.36(\mathrm{P}=0.03)$. Another important finding was positive correlation of $J A K 2 \mathrm{~V} 617 \mathrm{~F}$ expression with raised LDH and splenomegaly as shown in table-1 $(\mathrm{P} \leq 0.05)$. The mean splenic span was $21.48 \pm 2.96 \mathrm{~cm}$ in $J A K 2$ positive patients compared with negative patients having span of $15.80 \pm 3.37 \mathrm{~cm}(\mathrm{P}=0.05)$.

\section{Discussion}

Primary myelofibrosis is an uncommon hematopoietic malignancy with poorest prognosis of all myeloproliferative neoplasms (Martí-Carvajal et al., 2015). A distinctive feature of PMF is dysregulation of the Janus kinase (JAK)/ signal transducer and activator of transcription signaling pathway, particularly associated with the JAK2 V617F mutation, accountable in around $50 \%$ of patients with PMF (Stein et al., 2014).

In the present study, JAK2 V617F mutation was evaluated in Pakistani patients with PMF. It was noted that the prevalence of $J A K 2 \mathrm{~V} 617 \mathrm{~F}$ mutation in our patients from southern Pakistan was analogous with that was reported in the previous local study $(50 \%)$ from Northern Pakistan (Sadiq et al., 2013).

JAK2 V617F positivity in PMF has been observed from various ethnic backgrounds ranging from as low as $15 \%$ to as high as $76 \%$ (Poopak et al., 2013; Arana et 
al., 2014).

When compared with earlier reports, our results are in concurrence with regional studies reported from India; $58.8 \%$ and $52 \%$ for JAK2V617F mutational expression (Sazawal et al., 2010; Singh et al., 2015).

Other studies from Egypt and Romania by Ayad and Tevet et al have reported the frequency of JAK-2 positivity in $46 \%$ and $53.4 \%$ of patients with PMF respectively (Ayad and Nafea., 2011; Tevet et al., 2015). Another recent study from Thailand interestingly reported $100 \%$ positivity for JAK-2 mutation in Thai patients (Duangnapasatit et al., 2015). This high prevalence could be attributed to very small number (6 patients) of patients in their series (Duangnapasatit et al., 2015). However Zhang et al from China disclosed relatively low frequency for JAK-2 was detected in $40 \%$ of Chinese patients with PMF (Zhang et al., 2015).

Compared with data from developed countries outcome are more or less similar. Recent study from Taiwan also reported the similar prevalence $(50 \%)$ in their patients that is comparable to our findings (Chen et al., 2015). Subsequently Cross from United Kingdom also reported mutational frequency as $60 \%$ in a large cohort of patients (Cross, 2011). Mutation frequency was determined as $54 \%$ for $J A K 2 \mathrm{~V} 617 \mathrm{~F}$ in recent large series from the Italian group (Tefferi et al., 2014).

$J A K 2$ mutational expression clearly segregates the disease spectrum into two broad categories. It has been also reported previously that PMF cases with JAK-2 positivity displays a higher leucocyte count and haematocrit value as compared with negative phenotype. These concerns suggest that JAK-2 expression reflect a continued activation state of the stem cell malignant clone.

One study from Egypt reported significant correlation between high TLC counts and JAK-2 mutation (Ayad and Nafea., 2011). In the present study, significantly high total leukocytic count of $22.26 \pm 11.73$ was seen in JAK2 V617F positive patients groupn as compared with negative group that was $14.26 \pm 9.36(\mathrm{P}=0.03)$. In parallel to our findings, later studies from India, Iran and Turkey also demonstrated significant correlation of $J A K 2 \mathrm{~V} 617 \mathrm{~F}$ with raised total leukocytic count (Sazawal et al., 2010; Poopak et al., 2013; Singh et al., 2015; Yonal-Hindilerden et al., 2015).

However in conflict no correlation was established with high hemoglobin and JAK2 mutation in the present study. This variation represent high disease burden in our patients as $65 \%$ of our patients were anemic and it could have been due to the late presentation, avoidance of medical consultation and ignorance of general health practices.

However, marked splenomegaly is determined as a significant finding correlated with JAK-2 mutation in our study $(\mathrm{P}=0.05)$. In the available literature, we came across with the similar finding as reported in the recent Chinese study (Wang et al., 2014).

Lastly raised serum lactate dehydrogenase was also strongly correlated with JAK-2 mutation in our study. The mean LDH was $840.0 \pm 26.7$ in JAK-2 positive patients compared with $622 \pm 33.7$ in negative group $(\mathrm{P}=0.02)$. Likely, Larsen et al from Denmark also disclosed a strong association of $J A K 2$ mutation with high lactate dehydrogenase which is in concurrence to our results (Larsen et al., 2007).

In conclusion, our findings are analogous to studies reported from various part of world. Marked splenomegaly, high TLC and raised LDH levels indicate that our patients had clinically advanced disease. More so is JAK2 V617F mutated patients pointing an aggressive disease phenotype. We support the recommendation that mutational screening for $J A K 2 \mathrm{~V} 617 \mathrm{~F}$ should be incorporated into the initial evaluation of patients with suspected PMF.

\section{References}

Arana Yi C, Jeyakumar G, Medina P, et al (2014). Discrepancy in diagnosis of primary myelofibrosis between referral and tertiary care centers. Leuk Res, 38, 91-4.

Ayad MW, Nafea D (2011). Acquired mutation of the tyrosine kinase $J A K 2 \mathrm{~V} 617 \mathrm{~F}$ in Egyptian patients with myeloid disorders. Genet Test Mol Biomarkers, 15, 17-21.

Baxter EJ, Scott LM, Campbell PJ, et al (2005). Acquired mutation of the tyrosine kinase $J A K 2$ in human myeloproliferative disorders. Lancet, 365, 1054-61.

Barosi G, Bergamaschi G, Marchetti M, et al (2007). Gruppo Italiano Malattie Ematologiche Maligne dell'Adulto (GIMEMA) Italian Registry of Myelofibrosis. JAK2 V617F mutational status predicts progression to large splenomegaly and leukemic transformation in primary myelofibrosis. Blood, 110, 4030-6.

Chen YY, Huang CE, Lee KD, Chen CC (2015). Clinical efficacy and safety of ruxolitinib in the management of myelofibrosis: A single institution experience in Taiwan. Hematol. [Epub ahead of print]

Cross NC (2011). Genetic and epigenetic complexity in myeloproliferative neoplasms. Hematol Am Soc Hematol Educ Program, 2011, 208-14.

Duangnapasatit B, Rattarittamrong E, Rattanathammethee T, et al (2015). Clinical Manifestations and Risk Factors for Complications of Philadelphia Chromosome-Negative Myeloproliferative Neoplasms. Asian Pac J Cancer Prev, 16, 5013-8.

James C, Ugo V, Le Couedic JP, et al (2005). A unique clonal $J A K 2$ mutation leading to constitutive signalling causes polycythaemia vera. Nature, $\mathbf{4 3 4}, 1144-8$

Kim BH, Cho YU, Bae MH, et al (2015). JAK2 V617F, MPL, and CALR mutations in korean patients with essential thrombocythemia and primary myelofibrosis. J Korean Med Sci, 30, 882-8.

Larsen TS, Pallisgaard N, Moller MB, Hasselbalch HC (2007). The JAK2 V617F allele burden in essential thrombocythemia, polycythemia vera and primary myelofibrosis--impact on disease phenotype. Eur J Haemato, 79, 508-15.

Liu Y, Liu C, He N, et al (2015). JAK2 V617F mutation burden and its clinical implications in 415 patients with myeloproliferative neoplasm. Zhonghиа Хие Үе Хие Za Zhi, 36, 91-5.

Martí-Carvajal AJ, Anand V, Solà I (2015). Janus kinase-1 and Janus kinase-2 inhibitors for treating myelofibrosis. Cochrane Database Syst Rev, 10, 4.

Mesa RA, Silverstein MN, Jacobsen SJ, Wollan PC, Tefferi A (1999). Population-based incidence and survival figures in essential thrombocythemia and agnogenic myeloid metaplasia: an Olmsted County Study, 1976-1995. Am J Hematol, 61, 10-15.

Mitra D, Kaye JA, Piecoro LT, et al (2013). Symptom burden and splenomegaly in patients with myelofibrosis in the United States: a retrospective medical record review. Cancer Med, 


\section{2, 889-98.}

Poopak B, Hagh MF, Saki N, et al (2013). JAK2 V617F mutation in Iranian patients with myeloproliferative neoplasms: clinical and laboratory findings. Turkish J Med Sci, 43, 347-53.

Sadiq MA, Ahmed S, Ali N (2013). Frequency of Janus associated kinase 2 (JAK2) mutation in patients of BCRABL negative myeloproliferative neoplasms. Applied Life Sci, 2, 235-40.

Sag SO, Gorukmez O, Ture M, et al (2015). MMP2 gene-735 C/T and MMP9 gene $-1562 \mathrm{C} / \mathrm{T}$ polymorphisms in JAK2V617F positive myeloproliferative disorders. Asian Pac J Cancer Prev, 16, 443-9.

Sazawal S, Bajaj J, Chikkara S, et al (2010). Prevalence of $J A K 2$ V617F mutation in Indian patients with chronic myeloproliferative disorders. Indian J Med Re, 132, 423-7.

Stein BL, Swords R, Hochhaus A, Giles F (2014). Novel myelofibrosis treatment strategies: potential partners for combination therapies. Leukemia, 28, 2139-47.

Singh N, Sazawal S, Upadhyay A, et al (2015). Correlation of $J A K 2 \mathrm{~V} 617 \mathrm{~F}$ mutational status in primary myelofibrosis with clinico-hematologic characteristics and international prognostic scoring system scoring: a single center experience. Indian J Pathol Microbiol, 58, 187-91.

Tefferi A (2000). Myelofibrosis with myeloid metaplasia. N Engl J Med, 342, 1255-65.

Tefferi A, Guglielmelli P, Larson DR, et al (2014). Long-term survival and blast transformation in molecularly annotated essential thrombocythemia, polycythemia vera, and myelofibrosis. Blood, 124, 2507-13.

Thiele J, Kvasnicka HM, Orazi G, Tefferi A, Vardiman JM. Primary myelofibrosis. In: Swerdlow HS, Campo E, Haris LN, et al (2008). WHO classification of tumours of haemopoietic and lymphoid tissues. Lyon: International agency for research on cancer, 44.

Tevet M, Ionescu R, Dragan C, Lupu AR (2015). Influence of the JAK2 V617F Mutation and Inherited Thrombophilia on the Thrombotic Risk among Patients with Myeloproliferative Disorders. Mae, 10, 27-32.

Wang J, Xu J, Gale RP, et al (2014). Prognostic impact of splenomegaly on survival of Chinese with primary myelofibrosis. Leuk Res, 38, 1207-11.

Yang JJ, Chen H, Zheng XQ, et al (2015). Methylated alteration of SHP1 complements mutation of JAK2 tyrosine kinase in patients with myeloproliferative neoplasm. Asian Pac $J$ Cancer Prev, 16, 2219-25.

Yonal-Hindilerden I, Daglar-Aday A, Akadam-Teker B, et al (2015). The Burden of JAK2V617F Mutated Allele in Turkish Patients With Myeloproliferative Neoplasms. J Clin Med Res, 7, 161-70.

Zhang SP, Li H, Lai RS (2015). Detection of JAK2 V617F mutation increases the diagnosis of myeloproliferative neoplasms. Oncol Lett, 9, 735-8. 